\title{
Infectious Keratitis Secondary to Histoplasma capsulatum: The First Case Reports in Humans
}

\author{
Enyr S. Arcieri ${ }^{1,2}$, Ademir Rocha ${ }^{3}$, Camila N. Mendonça ${ }^{1}$, Eduardo G. V. Andreo ${ }^{1}$, Ioná G.A. Finotti ${ }^{1}$, Rafael L. Furlanetto ${ }^{1}$, Rafael S. $^{2}$ \\ Arcieri $^{1}$, Flávio J. Rocha ${ }^{1}$ and Luiz V. Rizzo ${ }^{4}$ \\ ${ }^{I}$ Department of Ophthalmology - School of Medicine - Federal University of Uberlândia (UFU); Uberlândia, MG; ${ }^{2}$ Department of \\ Ophthalmology - Glaucoma Service - University of Campinas (UNICAMP); Campinas, SP; ${ }^{3}$ Department of Pathology - School of Medicine - \\ Federal University of Uberlândia (UFU); Uberlândia, MG; ${ }^{4}$ Department of Immunology - Biomedical Sciences Institute - University of São \\ Paulo (USP); São Paulo, SP, Brazil
}

\begin{abstract}
The authors report an unusual case of fungal keratitis caused by Histoplasma capsulatum in a male immunocompetent patient. PCR confirmed the presence of the fungus DNA in the material studied. To our knowledge this is the first reported case in humans described all over the world. Key-Words: Human, keratitis, histoplasma, diagnosis.
\end{abstract}

Histoplasma capsulatum is a dimorphic unencapsulated fungus endemic in tropical areas including southeastern and central regions of the United States of America, West Indies, many parts of Central and South America, Africa, Asia and India [1].

Within the region of $H$. capsulatum endemicity in North America, there is a well-described syndrome attributed to infection with this fungus. This entity is known as presumed ocular histoplasmosis (POH), which occurs in immunocompetent individuals and is recognized by the presence of multiple disciform atrophic chorioretinal scars without vitreous or aqueous humor inflammation [2]. Active endophthalmitis in patients with disseminated histoplasmosis secondary to AIDS or immunosuppression may also occur [3].

Knox et al. [4] reported one case where a conjunctival granuloma was found to contain microorganisms compatible with $H$. capsulatum, confirmed by a fluorescent antibody technique, in a patient whose recent symptoms suggested systemic histoplasmosis. Thompson III et al. [5] described one case of histoplasmal conjunctivitis in an 82-year-old woman who complained of red eyes and had diagnosis of chronic sclerokeratitis.

To the best of our knowledge, there are no published reports that describe human cases of fungal keratitis due to H. capsulatum. Recent literature reports only one case of fungal keratitis due to Histoplasma spp. in a horse [6]. The purpose of this paper is to report the first case of fungal keratitis caused by $H$. capsulatum committing a human being all over the world.

\section{Case Report}

A 44-year-old white male was presented at the Ophthalmology Service - Federal University of Uberlândia

Received on 21 August 2007; revised 28 October 2007.

Address for correspondence: Dr. Enyr S. Arcieri. Rua Corcovado, n 155, ap 402, Uberlândia - Minas Gerais - Brazil. Zip code: 38411-092. Email: rsaran@triang.com.br. Phone : 55-34-32382400 / Fax: 55-3432384466. Presented as poster at 2006 ARVO Annual Meeting - Fort Lauderdale - Florida - USA.

The Brazilian Journal of Infectious Diseases 2007;11(6):595-597. (C) 2007 by The Brazilian Journal of Infectious Diseases and Contexto Publishing. All rights reserved.
(Brazil) on February 06, 2005, for an irritated and painful right eye (OD) of one month duration. He complained that the symptoms had worsened and started with photophobia and yellowish discharge three days before.

He reported outdoor activities and usually slept in caves. Although he denied previous systemic and ocular diseases or ocular trauma, his information was not reliable. His ophthalmologic exam showed vision corrected to hand motion OD and slit-lamp examination disclosed presence of abundant thick mucopurulent discharge, conjunctival hyperemia 4+/4+, a grayish stromal infiltrate with corneal thinning and a widespread epithelial defect.

Corneal scrapings were obtained aseptically from the base, leading edge and margins of the ulcer, using a flamesterilized Kimura's spatula. A portion of the scrapings were examined microscopically after digestion in $10 \% \mathrm{KOH}$ and in Gram-stained smears. The remaining material was inoculated onto blood-agar, chocolate-agar, Sabouraud's dextrose agar and thioglycolate broth. After obtained the scrapings, the patient was empirically started on topical $0.5 \%$ moxifloxacin and $5 \%$ fortified cefalotin every hour and $1 \%$ atropine thrice daily.

The culture grew Serratia marcescens and Streptococcus viridans resistant to cefalotin. The fortified cefalotin was discontinued and the patient continued with topical moxifloxacin and atropine. Despite this treatment, the patient presented decrease in his visual acuity to light perception, hypopyon and worse of corneal thinning. He underwent a therapeutic penetrating keratoplasty procedure on February 11 , and the corneal button had been sent to anatomopathologic exam. The corneal button specimen was formalin-fixed, paraffin-processed, and stained with routine hematoxylin-eosin stain, and methenamine silver stain.

On February 15 the results of anatomopathologic examination revealed acute fungal keratitis with a great quantity of intra- and extracellular globular structures, up to 4 $\mu \mathrm{m}$ in size, consisting of a central spherical deeply basophilic body surrounded by an unstained halo (Figures 1 and 2). The structures were morphologically consistent with $H$. capsulatum. The patient was started on a treatment of topical $0.5 \%$ amphotericin B drops hourly associated with topical moxifloxacin. 
Figure 1. Corneal stroma infiltrated with mononuclear cells and granulocyte neutrophil (stain, hematoxylin-eosin; original magnification, $\mathrm{x} 200$ ).

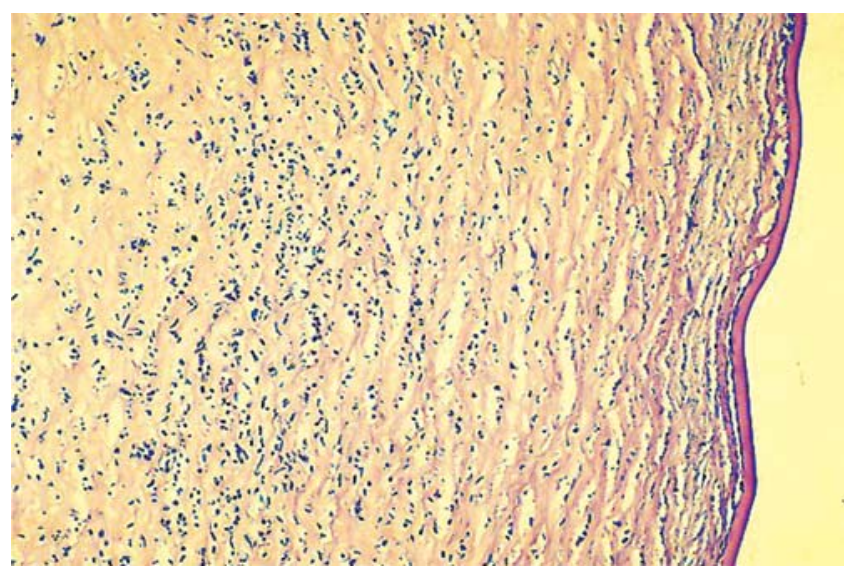

Topical $1 \%$ predinisolone acetate every six hours with gradual withdrawal was associated on March $1^{\text {st }}$, because the patient presented no signs of infection activity. Topical amphotericin B was discontinued on March 10. On June 13, he was using topical moxifloxacin and prednisone acetate every 12 hours. He remained asymptomatic, his visual acuity was hand motion and the cornea had no signs of infection recurrence. The patient was subsequently lost to follow up.

\section{Discussion}

Histoplasma capsulatum is a dimorphic fungus that remains in a mycelial form at ambient temperature and grows as yeast at body temperature in mammals [1]. H. capsulatum in the saprobic state grows in the mycelial form. Macroconidia and microconidia are produced on the hyphae of mycelium and are converted to the yeast form under temperaturecontrolled regulation. The aerosolization of conidia and mycelial fragments from contaminated soil results in alveolar deposition via inhalation. In the lung, the spores are ingested by macrophages and become yeasts. Most individuals who are infected are asymptomatic. Those who develop clinical manifestations are usually immunocompromised or are exposed to a high quantity of inoculum [1].

Approximately $1 \%$ to $10 \%$ of individuals living in endemic areas have ocular involvement as $\mathrm{POH}$, that is usually asymptomatic, but macula involvement may result in blindness [7]. Other eye changes in humans associated with $H$. capsulatum infection are endophthalmitis, conjunctival granuloma or conjunctivitis [4,5,8]. According to Gonzalez et al. [8], multiple factors may play a role in the clinical appearance and outcome of ocular histoplasmosis, including the virulence of the organism, size of the inoculum, and the resistance of the host.

Presumed ocular histoplasmosis is said to affect 2,000 new individuals a year in areas of endemicity and in some cases may lead to visual loss and blindness [7]. The lesions are thought to arise from hematogenous spread of the fungus
Figure 2. Corneal stroma with small budding yeast consistent with $H$. capsulatum (stain, methenamine silver; original magnification, $\mathrm{x} 400)$.

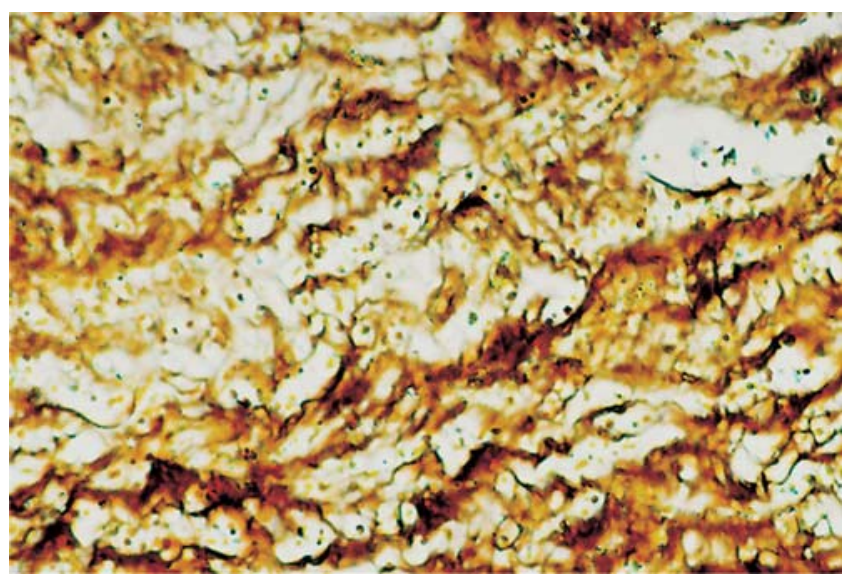

Figure 3. Lane 1-material from the eye; lane 2-material from a lung sample known to contain $H$. capsulatum; lane 3 negative control.

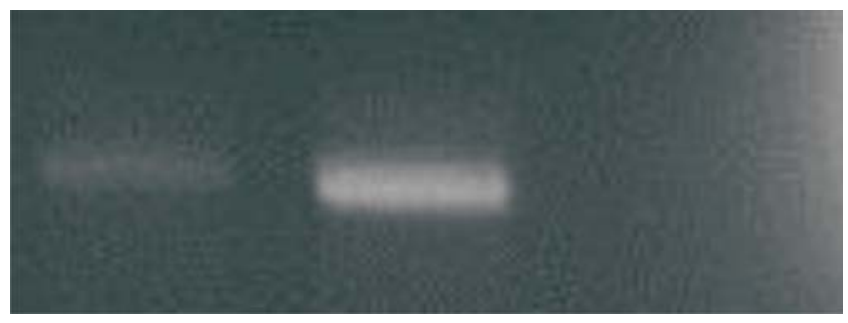

following initial infection and $H$. capsulatum is not detectable in the scars of $\mathrm{POH}$ [2], although products of $H$. capsulatum DNA were identified in histological sections from the enucleated eye of a patient with chronic $\mathrm{POH}$ [9].

Active endophthalmitis in patients with disseminated histoplasmosis secondary to AIDS or immunosuppression occurs and is associated with numerous budding yeast cells in the choroidal tissue and endothelium. In some cases the endophthalmitis is accompanied by yeast cells in the anterior chamber angle structures such as the iris, ciliary muscle and Schlemm's canal [3].

Although keratitis (corneal infection) is the most frequent presentation of ocular fungal infection [10], there are no published reports on literature describing human cases of keratitis due to $H$. capsulatum.

Mycotic keratitis is an important ophthalmologic problem all around the world, because, despite advances in diagnostic methods and medical treatment, it still causes visual disability. Regarding reports available in the literature from different parts of the world, it is evident that the incidence of fungal keratitis ranges from $6 \%$ to $56 \%$ [10]. According to some authors, $20 \%$ to $25 \%$ of fungal keratitis cases required therapeutic penetrating keratoplasty [10,11]. 
The most common cause of fungal keratitis is Fusarium solani and other Fusarium species, besides Aspergillus and Curvularia species [10]. The abnormal cornea in patients with dry eye syndrome, chronic ulceration, erythema multiform, and perhaps immunodeficiency virus (HIV) infection is subject to fungal infection, most commonly with Candida species [2].

Fungi are normally present as commensals in conjunctival flora of healthy eyes in about $3 \%$ to $28 \%$ and can invade the cornea in suitable conditions such as following trauma, corticosteroid administration, or in diseased corneas. Although the majority of fungal keratitis occurs after trauma to the cornea, the trauma may be so slight as to be forgotten by the patient [3]. According to Thomas [12], even the trivial trauma of a dust particle falling on the cornea may disrupt the integrity of the corneal epithelium, predisposing to mycotic keratitis.

The probable scenario of the case reported here is that fungal conidia from the environment, which could colonize the conjunctival sac as innocuous commensals, possibly turned pathogenic and invaded corneal tissue after brakes in the corneal epithelium.

To confirm the presence of $\mathrm{H}$. capsulatum DNA in our case, part of the paraffin embedded sample was cut and deparaffinized. DNA was extracted using DNAzol ${ }^{\mathrm{TM}}$ and prepared for PCR amplification according to the protocol described by Spencer et al. [9]. Samples were amplified by two methods, using as control a lung fragment known to contain the fungus. Extracted DNA was presented at all amplification solutions at a minimal of $100 \mathrm{ng}$.

Because the band intensity in the first set of primers used was not ideal and although similar band intensity has been considered positive previously [9], we felt compelled to confirm our results using primers designed and shown to amplify DNA from Brazilian strains of $H$. capsulatum (Figure 3 ). The results confirmed the presence of the fungus DNA in the material studied.

Regarding the presence of bacteria in the corneal scraping culture, some authors $[10,11]$ reported, in epidemiological studies, an incidence of up to $15.9 \%$ of coexistence between fungal and bacterial keratitis. There are two hypotheses that could explain the mixed fungal and bacterial corneal infection observed in this case: 1) the patient had an initial bacterial keratitis that predisposed to a secondary fungal infection; or 2) the patient presented a disruption in the integrity of the corneal epithelium, predisposing the fungal penetration in the cornea, causing a mycotic keratitis associated to a secondary bacterial infection.

In the present case, topical use of moxifloxacin and amphotericin B associated with therapeutic penetrating keratoplasty was successful in controlling the keratitis.

\section{References}

1. Davies S.F. Histoplasmosis: update 1989. Semin Respir Infect 1990;5:93-104.

2. Klotz S.A., Penn C.C., Negvesky G.J., Butrus S.I. Fungal and parasitic infections of the eye. Clin Microbiol Rev 2000; $13: 662-85$.

3. Font R.L., Parsons M.A., Keener M.J., et al. Involvement of anterior chamber angle structures in disseminated histoplasmosis: report of three cases. Ger J Ophthalmol 1995; 4 : $107-15$.

4. Knox D.L., O'Brien T.P., Green W.R. Histoplasma granuloma of the conjunctiva. Ophthalmology 2003;110:2051-3.

5. Thompson G.R. 3rd, LaValle CE 3rd, Everett ED. Unusual manifestations of histoplasmosis. Diagn Microbiol Infect Dis. 2004; 50:33-41.

6. Richter M., Hauser B., Kaps S., Spiess B.M. Keratitis due to Histoplasma spp. in a horse. Vet Ophthalmol 2003;6:99-103.

7. Magie S.K. Ocular histoplasmosis. In Fraunfelder FT, Roy FH, ed. Current ocular therapy. Philadelphia: W. B. Saunders Company; 1995, p. 75-7.

8. Gonzales C.A., Scott I.U., Chaudhry N.A., et al. Endogenous endophthalmitis caused by Histoplasma capsulatum var. capsulatum: a case report and literature review. Ophthalmology 2000; $107: 725-9$.

9. Spencer W.H., Chan C.C., Shen D.F., Rao N.A. Detection of Histoplasma capsulatum DNA in lesions of chronic ocular histoplasmosis syndrome. Arch Ophthalmol 2003;121:1551-5.

10. Srinivasan R., Kanungo R., Goyal J.L. Spectrum of oculomycosis in South India. Acta Ophthalmol (Copenh) 1991;69:744-9.

11. Vemuganti G.K., Garg P., Gopinathan U., et al. Evaluation of agent and host factors in progression of mycotic keratitis: A histologic and microbiologic study of 167 corneal buttons. Ophthalmology 2002; 109:1538-46.

12. Thomas P.A. Current perspectives on ophthalmic mycoses. Clin Microbiol Rev 2003;16:730-97. 